\title{
APPLICATION OF HPM TO FIND ANALYTICAL SOLUTION OF COETTE FLOW WITH VARIABLE VISCOSITY
}

\author{
Alireza AZIMI*, Mohammadreza AZIMI ${ }^{\star *}$, Amirhossein JAVANFAR $^{\star \star \star}$ \\ *Department of Chemical Engineering, College of Chemical Engineering, Islamic Azad University, \\ Mahshahr Branch, Mahshahr, Farhangsara Street, Iran \\ ${ }^{* *}$ Faculty of New Sciences and Technologies, Department of Aerospace, University of Tehran, Tehran, North Kargar, Amirabad, Iran \\ ${ }^{* * *}$ Faculty of Mechanical Engineering, Babol University of Technology, Shariati Street, Babol, Iran \\ meysam.azimi@gmail.com, m_r_azimi1991@yahoo.com, javanfar@ut.ac.ir
}

\begin{abstract}
In this paper, the couette flow of fluid with variable viscosity is studied analytically by using Homotopy Pertubation Method (HPM). At first the basic idea of Homotopy Pertubation Method (HPM) is presented. The mathematical formulation and application of HPM to nonlinear problem are presented in section three. In order to check the validity of solution the analytical results are compared with exact ones for various numerical cases. The good agreement between exact method and Homotopy Pertubation Method has been assures us about the solution accuracy.
\end{abstract}

Key words: Analytical Solution, HPM, Coette Flow, Viscosity

\section{INTRODUCTION}

Modelling of natural phenomena such as coquette flow and other fluid problems mostly leads to solving nonlinear equations. Except a limited number of these problems, most of them do not have analytical solution. So, the study on the various methods used for solving the nonlinear differential equations is a significant topic for the analysis of engineering problems. In recent years many powerful methods have been presented to construct solutions of Nonlinear Differential Equations such as Variational Iteration Method (VIM) (He, 1999), Homotopy Analysis Method (HAM) (Abasbandy, 2006), Homotopy Perturbation Method (Ghotbi et al., 2011; Jalal et al., 2011; Moghimi et al., 2011; Rashidi et al., 2012; Sharma and Methi, 2010) and Adomian Decomposition Method (Alan and Alkhaled, 2006; Ghosh, 2007; Lesnic, 2005; Pamuk, 2005).

The purpose of this present work is to present approximate analytical solution to a couette flow problem (Aziz and $\mathrm{Na}$, 1984) with variable viscosity by Homotopy perturbation method. In order to investigate the validity and accuracy of results, the obtained results are compared with the exact solution.

\section{BASIC IDEA OF HPM}

The homotopy perturbation method (HPM) was first proposed by He. The HPM does not depend upon a small parameter in the equation. By the homotopy technique in topology, a homotopy is constructed with an imbedding parameter $p \in[0,1]$, which is considered as a "small parameter".

The HPM was successfully applied to the nonlinear oscillators with discontinuities (He, 2004b) bifurcation of nonlinear problems
(He, 2004a), nonlinear wave equations (He, 2005). In (He, 2004a) comparison of HPM and homotopy analysis method was made, revealing that the former is more powerful than the later.

To illustrate the basic ideas of this method, we consider the following nonlinear differential equation (He, 2004a):

$A(u)-g(r)=0, r \in \Omega$

with boundary conditions:

$B(u, \partial u / \partial n)=0, r \in \Gamma$

where: $A, B, g(r)$ and $\Gamma$ are a general differential operator, a boundary operator, a known analytical function, and the boundary of domain $\Omega$.

Generally speaking the operator $A$ can be divided into a linear part $L$ and a nonlinear part $N(u)$. Equation (1), therefore, can be rewritten as follows:

$L(u)+N(u)-g(r)=0$

By the homotopy technique, we construct a homotopy $f(r, p): \Omega \times[0,1] \rightarrow R$ which satisfies:

$H(f, p)=(1-p)\left[L(f)-L\left(u_{0}\right)\right]+$ $p[A(f)-g(r)]=0, p \in[0,1], r \in \Omega$

or

$H(f, p)=L(f)-L\left(u_{0}\right)+p L\left(u_{0}\right)+$ $p[N(f)-g(r)]=0$

where $p \in[0,1]$ is an imbedding parameter, $u_{0}$ is an initial approximation of Equation (1), which satisfies the boundary conditions. Obviously, from Equations (4) and (5) we have:

$H(v, 0)=L(v)-L\left(u_{0}\right)=0$
$H(v, 1)=A(u)-f(r)=0$ 
the changing process of $p$ from zero to unity is just that of $v(r, p)$ from $u_{0}(r)$ to $u(r)$. In topology, this is called deformation, and $L(v)-L\left(u_{0}\right)$ and $A(u)-f(r)$ are called homotopy.

According to the HPM, we can first use the imbedding parameter $p$ as a "small parameter", and assumed that the solution of Equations (4) and (5) can be written as a power series in $p$ :

$f=f_{0}+p f_{1}+p^{2} f_{2}+p^{3} f_{3}+\cdots$ (3):

Setting $p=1$ results in the approximate solution of Equation

$$
f=\lim _{p \rightarrow 1} f=f_{0}+f_{1}+f_{2}+\cdots
$$

The coupling of the perturbation method and the homotopy method is called the homotopy perturbation method (HPM), which has eliminated limitations of the traditional perturbation methods. In the other hand, this technique can take full advantage of the traditional perturbation techniques.

The series $(8,9)$ are convergent for most cases, however, the convergent rate depends upon the nonlinear operator $N(v)$. The following opinions are suggested by $\mathrm{He}(\mathrm{He}, 2004 \mathrm{a})$ :

1. The second derivative of $N(v)$ with respect to $v$ must be small, because the parameter $p$ may be relatively large, i.e. $p \rightarrow 1$.

2. The norm of $L^{-1}(\partial N / \partial v)$ must be smaller than one, in order that the series converges.

\section{SOLUTION PROCEDURE}

Consider the steady flow of an incompressible Newtonian fluid between two infinite, parallel plates separated by a distance $a$ as shown in Fig. 1. Each plate is maintained at temperature $T_{0}$. The lower plate is stationary while the upper plate moves with a uniform velocity $V$. The thermal conductivity of the fluid is assumed to be constant, but the viscosity is allowed to vary. The pertinent momentum and energy equations are(Aziz and $\mathrm{Na}, 1984)$ :

$\frac{d}{d y}\left(\mu \frac{d u}{d y}\right)=0$

$\frac{d^{2} T}{d y^{2}}+\frac{\mu}{k}\left(\frac{d u}{d y}\right)^{2}=0$

$y=0, \quad u=0, \quad T=T_{0}$

$y=a, \quad u=V, \quad T=T_{0}$

where: $u$ - axial velocity, $T$ - temperature, $\mu$ - viscosity and $k$ - thermal conductivity.

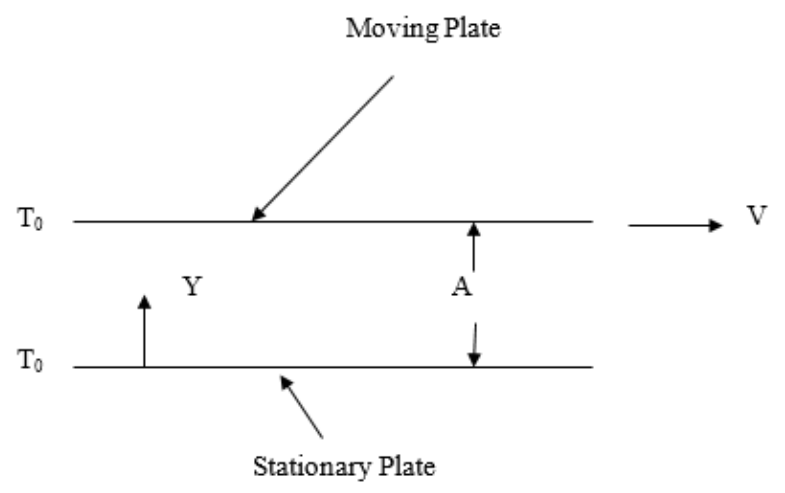

Let the viscosity vary exponentially with temperature according to:

$\mu=\mu_{0} e^{-\alpha\left(T-T_{0}\right)}$

where: $\mu_{0}$ is the viscosity at $T_{0}$ and $a$ is a constant.

Introduce Equation (14) and the following dimensionless quantities into Equations (10)-(13):

$$
\begin{gathered}
\theta=\frac{T-T_{0}}{T_{0}}, \quad Y=\frac{y}{a}, \quad U=\frac{u}{V} \\
\beta=\alpha T_{0}, \quad \varepsilon=\frac{\mu_{0} V^{2}}{k T_{0}}
\end{gathered}
$$

to give:

$\left\{\begin{array}{l}\frac{d}{d Y}\left(e^{-\beta \theta} \frac{d U}{d Y}\right)=0 \\ \frac{d^{2} \theta}{d x}+\varepsilon e^{-\beta \theta}\left(\frac{d U}{d Y}\right)^{2}=0\end{array}\right.$

$\left\{\begin{array}{l}Y=0, \quad U=0, \quad \theta=0 \\ Y=1, \quad U=1, \quad \theta=0\end{array}\right.$

$\{Y=1, \quad U=1, \quad \theta=0$

In this case, the parameter $\mu_{0} V^{2} / k T_{0}$ is identified as the perturbation quantity $\varepsilon$. In viscous flow terminology, it is called the Brinkman number, and represents the ratio of viscous heating due to conduction. Thus, if the effect of viscous heating is weak, one may treat $\varepsilon$ as small and carry out a perturbation analysis. As shown by Turian and Bird (1963), such an analysis is applicable to flow in a cone-and-plate viscometer.

In order to solve this system with homotopy perturbation method (HPM), we consider:

$U(Y)=v_{1}(Y), \quad \theta(Y)=v_{2}(Y)$

$v_{1,0}(Y)=U_{0}(Y), \quad v_{2,0}(Y)=\theta_{0}(Y)$

$v_{1}(Y)=v_{1,0}(Y)+p v_{1,1}(Y)+p^{2} v_{1,2}(Y)$

$v_{2}(Y)=v_{2,0}(Y)+p v_{2,1}(Y)+p^{2} v_{2,2}(Y)$

the term $e^{-\beta i \theta(Y)}$ must be expanded as follows:

$e^{-\beta \theta(Y)}=1-\beta \theta(Y)+\frac{1}{2} \beta^{2} \theta^{2}(Y)$

The substitution of Equation (20) into Equation (16) yields:

$\left\{\begin{array}{l}\left(1-\beta \theta+\frac{1}{2} \beta^{2} \theta^{2}\right) \frac{d^{2} U}{d Y^{2}}+\beta\left(\beta \theta-\frac{1}{2} \beta^{2} \theta^{2}-1\right) \frac{d U}{d Y} \frac{d \theta}{d Y}=0 \\ \frac{d^{2} \theta}{d Y^{2}}+\varepsilon\left(1-\beta \theta+\frac{1}{2} \beta^{2} \theta^{2}\right)\left(\frac{d U}{d Y}\right)^{2}=0\end{array}\right.$

According to the HPM, we must firstly determine the initial approximations for $v_{1}(Y)$ and $v_{2}(Y)$. So we construct following system for linear parts:

$\left\{\begin{array}{l}\frac{d^{2} U_{0}}{d Y^{2}}=0 \\ \frac{d^{2} \theta_{0}}{d Y^{2}}=0\end{array}\right.$

with following conditions:

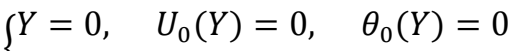

$\left\{Y=1, \quad U_{0}(Y)=1, \quad \theta_{0}(Y)=0\right.$

The solution of Equation (45) is as follows:

$\left\{\begin{array}{l}U_{0}(Y)=v_{1,0}(Y)=Y \\ \theta_{0}(Y)=v_{2,0}(Y)=0\end{array}\right.$

A homotopy for the system of Equation (21) can be constructed as follows:

Fig. 1. Plane Coette Flow 


$$
\left\{\begin{array}{l}
(1-p)\left(\frac{d^{2} v_{1}}{d Y^{2}}-\frac{d^{2} v_{1,0}}{d Y^{2}}\right)+ \\
p\left(\left(1-\beta v_{2}+\frac{1}{2} \beta^{2} v_{2}^{2}\right) \frac{d^{2} v_{1}}{d Y^{2}}\right)+\beta\left(\beta v_{2}-\frac{1}{2} \beta^{2} v_{2}^{2}-1\right) \frac{d v_{1}}{d Y} \frac{d v_{2}}{d Y}=0 \\
(1-p)\left(\frac{d^{2} v_{2}}{d Y^{2}}-\frac{d^{2} v_{2,0}}{d Y^{2}}\right)+ \\
p\left(\frac{d^{2} v_{2}}{d Y^{2}}+\left(1-\beta v_{2}+\frac{1}{2} \beta^{2} v_{2}^{2}\right) \varepsilon\left(\frac{d v_{1}}{d Y}\right)\right)=0
\end{array}\right.
$$

The substitution of Equation (24) into Equation (25) yields:

$$
\left\{\begin{array}{l}
(1-p)\left(\frac{d^{2} v_{1}}{d Y^{2}}\right)+ \\
p\left(\left(1-\beta v_{2}+\frac{1}{2} \beta^{2} v_{2}^{2}\right) \frac{d^{2} v_{1}}{d Y^{2}}+\beta\left(\beta v_{2}-\frac{1}{2} \beta^{2} v_{2}^{2}-1\right) \frac{d v_{1}}{d Y} \frac{d v_{2}}{d Y}\right)=0 \\
(1-p)\left(\frac{d^{2} v_{2}}{d Y^{2}}\right)+ \\
p\left(\frac{d^{2} v_{2}}{d Y^{2}}+\left(1-\beta v_{2}+\frac{1}{2} \beta^{2} v_{2}^{2}\right) \varepsilon\left(\frac{d v_{1}}{d Y}\right)\right)=0
\end{array}\right.
$$

The substitution of Equation (19) into Equation (26) and collect the result up to $p^{2}$ yields:

$$
\left\{\begin{array}{l}
\left(-\beta \frac{d v_{2,1}}{d Y}+\frac{d^{2} v_{1,2}}{d Y^{2}}\right) p^{2}+\frac{d^{2} v_{1,1}}{d Y^{2}} p=0 \\
\left(\frac{d^{2} v_{2,2}}{d Y^{2}}-\varepsilon \beta v_{2,1}\right) p^{2}+\left(\frac{d^{2} v_{2,1}}{d Y^{2}}+\varepsilon\right) p=0
\end{array}\right.
$$

then we must equalize the coefficients of $p$ and $p^{2}$ in Equation (27) with zero:

$$
\left\{\begin{array}{l}
\frac{d^{2} v_{1,1}}{d Y^{2}}=0 \\
\frac{d^{2} v_{2,1}}{d Y^{2}}+\varepsilon=0 \\
-\beta \frac{d v_{2,1}}{d Y}+\frac{d^{2} v_{1,2}}{d Y^{2}}=0 \\
\frac{d^{2} v_{2,2}}{d Y^{2}}-\varepsilon \beta v_{2,1}=0
\end{array}\right.
$$

with following conditions:

$v_{i, j}(0)=v_{i, j}(1)=0, \quad i, j=1,2$

therefore the results are as follows:

$$
\left\{\begin{array}{l}
v_{1,1}(Y)=0 \\
v_{1,2}(Y)=-\frac{1}{6} \beta \varepsilon Y^{3}+\frac{1}{4} \beta \varepsilon Y^{2}-\frac{1}{12} \beta \varepsilon Y \\
v_{2,1}(Y)=-\frac{1}{2} \varepsilon Y^{2}+\frac{1}{2} \varepsilon Y \\
v_{2,2}(Y)=-\frac{1}{24} \beta \varepsilon^{2} Y^{4}+\frac{1}{12} \beta \varepsilon^{2} Y^{3}-\frac{1}{24} \beta \varepsilon^{2} Y
\end{array}\right.
$$

According to the HPM, we can conclude:

$$
\begin{aligned}
& U(Y)=\lim _{p \rightarrow 1}\left(v_{1,0}(Y)+p v_{1,1}(Y)+p^{2} v_{1,2}(Y)\right) \\
& \theta(Y)=\lim _{p \rightarrow 1}\left(v_{2,0}(Y)+p v_{2,1}(Y)+p^{2} v_{2,2}(Y)\right)
\end{aligned}
$$

The substitution of Equations (24) and (30) into Equation (31) yields:

$$
\begin{aligned}
U(Y)= & Y-\frac{1}{2} \beta \varepsilon\left(\frac{1}{3} Y^{3}-\frac{1}{2} Y^{2}\right)-\frac{1}{12} \beta \varepsilon Y \\
\theta(Y)=-\frac{1}{2} \varepsilon Y^{2} & +\frac{1}{2} \varepsilon Y-\frac{1}{2} \beta \varepsilon^{2}\left(\frac{1}{12} Y^{4}-\frac{1}{6} Y^{3}\right) \\
& -\frac{1}{24} \varepsilon^{2} \beta Y
\end{aligned}
$$

We avoid listing the other components. The forgoing homotopy perturbation solution may be compared with the exact solution (Turian and Bird, 1963) which is given by:

$$
\begin{aligned}
& e^{\beta \theta}=\left(1+\frac{\varepsilon \beta}{8}\right) \operatorname{sech}^{2}\left[(2 Y-1) \sinh ^{-1}\left(\frac{\varepsilon \beta}{8}\right)^{1 / 2}\right] \\
& U=\frac{1}{2}\left\{\left(1+\frac{8}{\varepsilon \beta}\right)^{1 / 2} \tanh \left[(2 Y-1) \sinh ^{-1}\left(\frac{\varepsilon \beta}{8}\right)^{1 / 2}\right]+1\right\}
\end{aligned}
$$

\section{RESULTS AND DISCUSSIONS}

In previous section, analytical investigation of plane couette flow with variable viscosity is presented. In this section, we will present the obtained results. As it can be illustrated in Fig. 2 the comparison is made between HPM solution and exact ones for $\beta=1$ and a range of values of $\varepsilon$. In Fig. 3.the deviations with the exact solutions have been plotted for $\beta=1$ and a range of values of $\varepsilon$.

According to Fig. 3 effect of the Brinkman number on temperature profile is considerable. The increasing of Brinkman number cause higher peak in temperature profile.

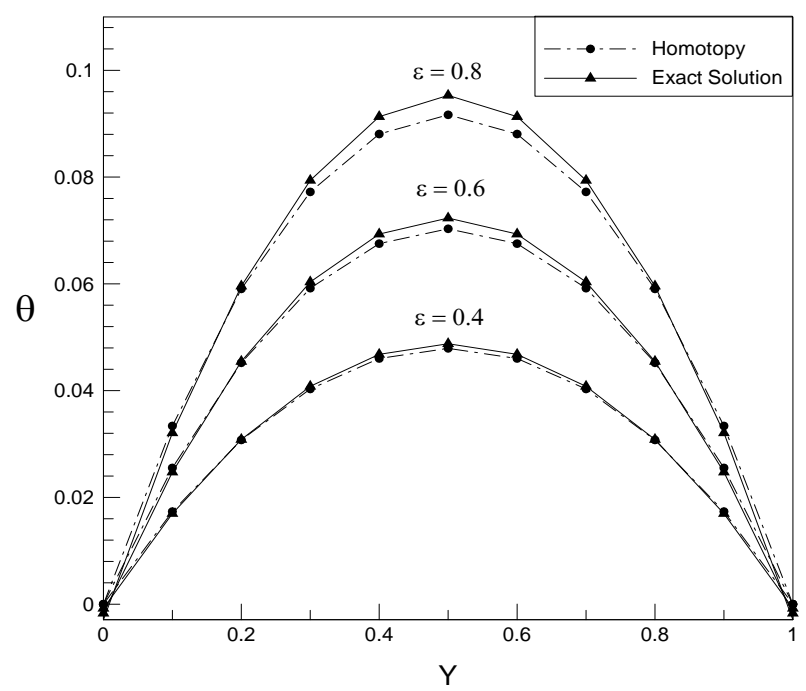

Fig. 2. Temperature distribution in plane couette flow, $\beta=1$

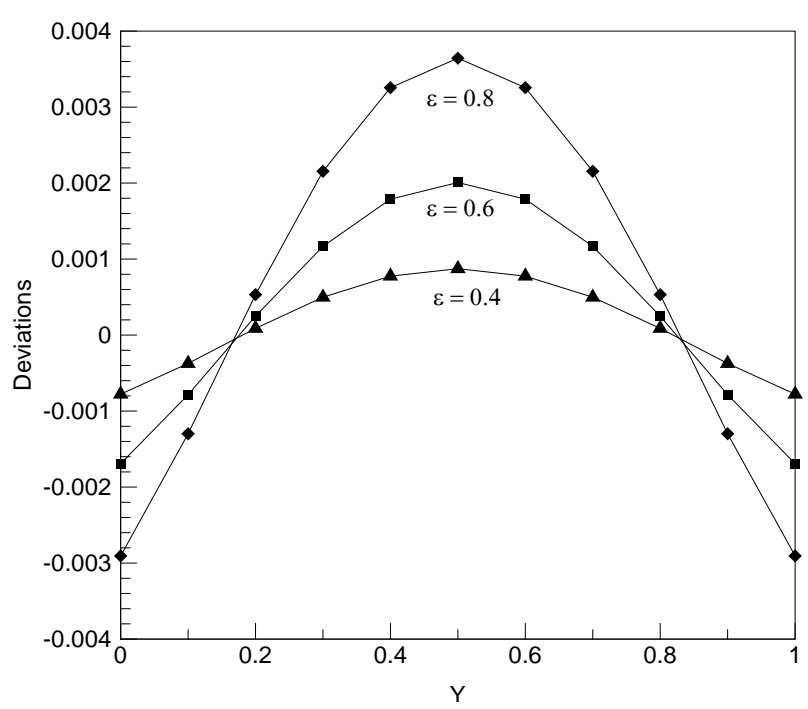

Fig. 3. Deviations of the HPM (2-term expansion) results with exact solution, with fixed value $\beta=1$ 
Tab. 1 shows the effect of increasing the number of HPM terms on obtained temperature values for a range of values of $\varepsilon$ when $Y=0.5, \beta=1$.

Tab. 1. Comparison between Exact solutions and Analytical results for various term expansions of HPM and different value of $\varepsilon$ in case $Y=0.5, \beta=1$

\begin{tabular}{|c|c|c|c|}
\hline$\varepsilon$ & $\begin{array}{c}\text { HPM } \\
\text { (3-term expansion) }\end{array}$ & $\begin{array}{c}\text { HPM } \\
\text { (4-term expansion) }\end{array}$ & Exact \\
\hline 0.05 & 0.0061514 & 0.0062104 & 0.0062305 \\
\hline 0.5 & 0.0604317 & 0.0606101 & 0.0606246 \\
\hline 1 & 0.1176017 & 0.117721 & 0.1177831 \\
\hline 1.5 & 0.1717675 & 0.1718101 & 0.1718502 \\
\hline 2 & 0.2210188 & 0.2230992 & 0.2231435 \\
\hline
\end{tabular}

Good agreement between analytical approximate solution and numerical method assure us about validity and accuracy of solution.

\section{CONCLUSIONS}

In this paper, the homotopy perturbation method (HPM) has been successfully applied to finding the solutions of plane couette flow with variable viscosity. The HPM does not require small parameters in the equations, so that the limitations of the traditional perturbation methods can be eliminated and the calculations in HPM is simple and straightforward. In HPM, the approximations obtained are valid, not only for small parameters, but also for larger ones. The results show that the HPM is a powerful mathematical tool for solving differential system, it is also a promising method to solve other nonlinear ordinary and partial differential systems. The solutions obtained are shown graphically and compared with the exact solution.

\section{REFERENCES}

1. Abbasbandy S. (2006), The application of homotopy analysis method to nonlinear equations arising in heat transfer, Physics Letter, A 360, 109-113.

2. Allan F., Al-Khaled K. (2006), An approximation of the analytic solution of the shock wave equation, Journal of Computational and Applied Mathematics, 192, 301-309.

3. Aziz A., Na T. Y. (1984), Perturbation methods in heat transfer, Hemisphere Publishing Corp.

4. Ghosh S., Roy A., Roy D. (2007), An adaptation of Adomian decomposition for numeric-analytic integration of strongly nonlinear and chaotic oscillators, Computer Methods in Applied Mechanics and Engineering, 196, 1133-1153.

5. Ghotbi A. R., Barari A. Ganji D. D. (2011), Solving ratio-dependent predator-prey system with constant effort harvesting using homotopy perturbation method, Mathematical Problems in Engineering, ID 945420.

6. He J. H. (1999), Variational iteration method: A kind of nonlinear analytical technique: Some examples, International Journal of NonLinear Mechanics, 344, 699-708.

7. He J. H. (2004a), Comparison of homotopy perturbation method and homotopy analysis method, Appl. Math Comput., 156, 527-39.

8. He J. H. (2004b), The homotopy perturbation method for nonlinear oscillators with discontinuities, Appl. Math Comput., 151, 287-92.
9. He J. H. (2005), Homotopy perturbation method forbifurcation of nonlinear problems, Int. J. Nonlinear Sci. Numer. Simul., 6(2), 207-215.

10. Jalaal M., Nejad M. G., Jalili P. (2011), Homotopy perturbation method for motion of a spherical solid particle in plane couette fluid flow, Computers and Mathematics with Applications, 61, 2267-2270.

11. Lesnic D. (2005), Decomposition methods for non-linear, noncharacteristic Cauchy heat problems, Communications in Nonlinear Science and Numerical Simulation, 10, 581-596.

12. Moghimi S. M., Ganji D. D., Bararnia H., Hosseini M., Jalaal M. (2011), Homotopy perturbation method for nonlinear MHD JefferyHamel problem, Computers and Mathematics with Applications, 61, 2213-2216.

13. Pamuk S. (2005), Solution of the porous media equation by Adomian's decomposition method, Physics Letters, A 344, 184-188.

14. Rashidi R. R., Beg O.A., Rastegari M.T., Mehmood A. (2012), Homotopy study of buoyancy-induced flow of non-newtonian fluids over a non-isothermal surface in a porous medium, International Journal of Applied Mathematics and Mechanics, 8, 34-52.

15. Sharma P. R., Methi G. (2010), Solution of coupled nonlinear partial differential equations using homotopy perturbation method, International Journal of Applied Mathematics and Mechanics, 6, 33-49.

16. Turian R. M., Bird R. B. (1963), Viscous heating in the cone-andplate viscometer II, Chem. Eng. Sci., 18, 689-96. 